\title{
Swift XRT Observations of the Possible Dark Galaxy VIRGOHI 21
}

\author{
Massimiliano Bonamente ${ }^{1,2}$, Douglas A. Swartz ${ }^{3}$, Martin C. Weisskopf ${ }^{2}$, and \\ Stephen S. Murray ${ }^{4}$
}

\begin{abstract}
Swift XRT observations of the Hi line source VIRGOHI 21 were performed on April 22 and April 26, 2008 for a total exposure time of $9.2 \mathrm{ks}$. This is the first pointed X-ray observation of VIRGOHI 21, a putative dark galaxy in the Virgo cluster, and no photons were detected from this source. The non-detection of extended X-ray emission within the angular extent of the Hi source corresponds to a $99 \%$ confidence upper limit of $2.1 \times 10^{-14} \mathrm{ergs} \mathrm{cm}^{-2} \mathrm{~s}^{-1}$ in the $0.3-2.0 \mathrm{keV}$ band. The equivalent upper limit to the amount of diffuse hot gas associated with VIRGOHI 21 is in the range $4 \times 10^{7}-2 \times 10^{8} M_{\odot}$ for a hot gas temperature between 0.1 and $1 \mathrm{keV}$. The non-detection also corresponds to a $99 \%$-confidence upper limit on the flux from a point-like source of $8 \times 10^{-15} \mathrm{ergs}^{-2} \mathrm{~s}^{-1}$ in the $0.3-2.0 \mathrm{keV}$ band. We discuss the constraints on the nature of VIRGOHI 21 imposed by these observations and the theoretical implications of these results.
\end{abstract}

Subject headings: dark matter — galaxies: individual (VIRGOHI 21) - X-rays: galaxies

\section{Introduction}

Hi surveys of the Virgo Cluster (Davies et al. 2004, 2006; Minchin et al. 2005, 2007; Haynes et al. 2007) have revealed a remarkable Hi cloud, VIRGOHI 21, with apparent evidence of rotation yet unaccompanied by any optical emission. The Hi mass $\left(M_{H I} \sim\right.$ $\left.3 \times 10^{7} M_{\odot}\right)$, inferred circular velocity $\left(V \sim 100 \mathrm{~km} \mathrm{~s}^{-1}\right)$ and physical size $(R \sim 8 \mathrm{kpc})$ are

\footnotetext{
${ }^{1}$ Department of Physics, University of Alabama, Huntsville, AL, USA

${ }^{2}$ NASA Marshall Space Flight Center, Huntsville, AL, USA

${ }^{3}$ Universities Space Research Association, NASA Marshall Space Flight Center, VP62, Huntsville, AL, USA

${ }^{4}$ Harvard-Smithsonian Center for Astrophysics, 60 Garden Street, MS-67, Cambridge, MA 02138
} 
all typical of luminous galaxies of dynamical masses $10^{10}-10^{11} M_{\odot}$ yet deep optical follow-up observations provide an upper limit of only $31.1 \mathrm{I} \mathrm{mag} \operatorname{arcsec}^{-2}$, corresponding to a stellar content of $2 \times 10^{5} L_{\odot}$ (Minchin et al. 2007). Thus, VIRGOHI 21 is currently considered the prototypical dark galaxy candidate: a dynamically-massive dark-matter-dominated galaxy with a rotating gaseous disk but devoid of stars.

If VIRGOHI 21 is a massive dark galaxy, then it has important implications for the theory of structure formation. The highly-successful cosmological hierarchical clustering model originated by White \& Rees (1978) is based on the idea of accretion and merging of cold dark matter $(\mathrm{CDM})$ from small sizes $\left(\sim 10^{8} M_{\odot}\right)$ up to the large galaxy clusters observed at the present epoch. The CDM model predicts a large number of $\gtrsim 10^{8} M_{\odot}$ dark matter (DM) halos should remain unassimilated following hierarchical growth and thus still be present today (e.g., Moore et al. 1999; Klypin et al. 1999; Davies et al. 2006). To date, however, the number of observed low-mass galaxies falls far short of these predictions. This is true locally, where only a handful of satellites surround the massive Local Group spirals (e.g., Mateo 1998), and is manifest in the faint end of the luminosity function that shows a slope more shallow than $\Lambda$ CDM predictions (Blanton et al. 2001; Benson et al. 2003). This shortcoming of the $\Lambda \mathrm{CDM}$ model can be overcome either by preventing small DM halos from forming in the first place or by suppressing star formation so that they remain optically faint. The latter possibility implies a population of low-mass DM halos in which the baryon content remains optically-dark but still visible chiefly through HI gas emission. The rarity of isolated extragalactic Hi clouds lacking optical counterparts (Doyle et al. 2005) suggests that VIRGOHI 21, if truly massive, is a rare case in favor of this scenario.

An alternative to the dark galaxy interpretation for VIRGOHI 21 (Bekki et al. 2005; Haynes et al. 2007; Duc \& Bournaud 2008) is that it is simply a part of an elongated HIdominated tidal tail emanating from the nearby luminous galaxy NGC 4254 (M99). The tidal tail was caused either by a fly-by encounter with another (massive) galaxy (Vollmer et al. 2005) or by ram pressure stripping within the Virgo environment. Recent observations show the Hi gas in the tail extends from NGC 4254, located $\sim 120 \mathrm{kpc}$ to the south of VIRGOHI 21, to about $130 \mathrm{kpc}$ beyond VIRGOHI 21 to the north (Haynes et al. 2007). The peculiar velocity structure in the vicinity of VIRGOHI 21 is, in this case, ascribed to projection effects on the streaming motions of the debris tail instead of to rotation within a gravitationally bound disk. In this scenario, the estimated Hi mass is close to the total mass of the object; there is no massive halo accompanying VIRGOHI 21.

In $\S 2$ we describe the Swift observations leading to upper limits of the X-ray emission from VIRGOHI 21, and in $\S 3$ we provide our interpretation of the Swift non-detection. In this paper we assume a distance to the source of $D=16.5 \mathrm{Mpc}$ corresponding to the mean 
of the Virgo Cluster (Mei et al. 2007) and a scale of $4.9 \mathrm{kpc}$ per arcminute.

\section{SWIFT observations of VIRGOHI 21 and data analysis}

The VIRGOHI 21 field was observed by Swift on 2008 April 22 and 26 with the XRT operated in photon counting mode. The XRT has a very low detector background, which makes it especially suitable for observations of faint diffuse X-ray sources. These data are the first pointed X-ray observations of VIRGOHI 21.

The data were screened for bad pixels and other detector artifacts, and only photon grades $0-12$ were included in the analysis. The screening resulted in $9.2 \mathrm{ks}$ of clean data. In this analysis we considered photon energies in the $0.3-7 \mathrm{keV}$ range, where the calibration of the XRT is better understood. The data reduction was performed using xselect and the FTOOLS, and the spectral analysis with XSPEC.

VIRGOHI 21 lies about $1 \mathrm{Mpc}$ to the NW of the center of the Virgo Cluster at R.A. $=12^{\mathrm{h}} 17^{\mathrm{m}} 53.6^{\mathrm{s}}$ decl. $=+14^{\circ} 45^{\prime} 25^{\prime \prime}(\mathrm{J} 2000.0)$. The angular size of VIRGOHI $21,3.5^{\prime} \times 1.375^{\prime}$, lies well within the $24^{\prime} \times 24^{\prime}$ field of view of the XRT (Cusumano et al. 2006; Romano et al. 2005). X-ray images of the VIRGOHI 21 field are shown in Figure 1, in which the gray contour are from the Hi observations of Minchin et al. (2007). The upper part of the HI feature was interpreted by Minchin et al. (2007) as a rotating disc, and the lower part as an Hi bridge connecting VIRGOHI 21 with NGC 4254, located to the south and outside the Swift field of view.

As with normal galaxies, VIRGOHI 21 was expected to be a source of both diffuse and point-source X-ray emission. Even in the absence of stars, gas falling into a galaxy's gravitational potential from the intergalactic medium should be heated to roughly the (Xray-emitting) virial temperature. In addition, accretion onto a central supermassive black hole from the gas reservoir in VIRGOHI 21, even at a fraction of the Bondi rate, should appear as a localized source of X-radiation though not necessarily optically bright. No X-ray emission associated with VIRGOHI 21 was detected. An unrelated weak (41 counts) pointlike source was detected near R.A. $=12^{h} 17^{m} 23^{s}$, decl. $=14^{\circ} 39^{\prime} 29^{\prime \prime}$ (Figure 1). As no object is known within $1^{\prime}$ of this location, we regard this as the discovery of the X-ray source SWIFTXRT J121723+1439.5. In the following, we place upper limits to the presence of diffuse and point source emission from VIRGOHI 21. 


\subsection{Diffuse X-ray emission}

In order to place upper limits to the diffuse soft X-ray emission from VIRGOHI 21, we accumulated X-ray photons from a $3.5^{\prime} \times 1.375^{\prime}$ region located at the position of the putative Hi disc (black box in Figure 1). We detected a total of 8 counts in the $0.3-2 \mathrm{keV}$ band, and no counts in the $2-7 \mathrm{keV}$ band. We estimate the number of background counts, first by extracting the data from the entire field of view, excluding a region of enhanced emission to the south (Figure 1) and regions close to the detector boundaries. The region included encompassed 337.3 square arcminutes. We found 623 counts in the $0.3-2 \mathrm{keV}$ band and 319 counts in the 2-7 keV band. Ignoring the possibility that some of these events might be vignetted by the telescope one therefore expects in the region of interest at least $8.9 \pm 0.4$ counts in the soft band and $4.6 \pm 0.3$ in the hard band. We note that it is mildly interesting and somewhat improbable that no counts were measured in the hard band from the region containing VIRGOHI 21.

We use the soft X-ray band data in order to set upper limits as to source detection, since this is the band in which the X-ray emission from diffuse gas (and point sources) is expected. Since the measured background predicts at least a mean of $8.9 \pm 0.4$ counts and we detected 8 counts it is therefore evident that the VIRGOHI 21 region is not an X-ray emitter. To determine upper limits we increased the expected number of counts in the soft band by $10 \%$ to 9.8 counts to account for the possibility that some of the measured background events arise from faint, unresolved X-ray sources. We determine 99\%-confidence upper limits assuming 18 counts as detection.

\subsubsection{Uniform distribution}

We assume that the Hi disk is seen edge-on, and thus the emitting volume is $V=\pi R^{2} L$, where $R=1.75$ ' and $L=1.375$ '. We use an optically-thin plasma of uniform density and of Solar abundance (apec in XSPEC), a Galactic HI column density of $N_{H}=2.7 \times 10^{20} \mathrm{~cm}^{-2}$ (Dickey \& Lockman 1990; Kalberla et al. 2005) with Morrison \& McCammon (1983) crosssections (wabs in XSPEC), and calculate the emission measure required to achieve 8.1 source counts as a function of plasma temperature using XSPEC. The emission measure calculated by XSPEC is given by the model normalization, $K$, as

$$
K=\frac{10^{-14}}{4 \pi D_{A}^{2}} \int n_{e} n_{H} d V,
$$

in which $n_{e}$ and $n_{H}$ are, respectively, the electron and hydrogen number density and $D_{A}$ the angular size distance (for $z \ll 1$ ). From this, we calculate the gas density, and thus the gas 
mass. The results are shown in Figure 2, showing that these 9.2 ks Swift XRT observations set upper limits of the order of $10^{8} M_{\odot}$. These upper limits correspond to a flux of $2.1 \times 10^{-14}$ ergs $\mathrm{cm}^{-2} \mathrm{~s}^{-1}$ in the $0.3-2 \mathrm{keV}$ band.

\subsection{2. $\beta$ model distribution}

A putative hot halo may be maintained in hydrostatic equilibrium by the gravitational potential of the galaxy. In this case, the gas density will decrease with radius. We therefore consider a more realistic model of the gas density given by the $\beta$ model,

$$
n_{e}=n_{e 0}\left(1+\frac{r^{2}}{r_{c}^{2}}\right)^{-\frac{3}{2} \beta}
$$

as often observed in galaxy clusters (Cavaliere \& Fusco-Femiano 1978). The parameters $r_{c}$ and $\beta$ describe, respectively, the radius within which the density is approximately constant, and the decrease of the density at large radii. For this interpretation, we use a spherically symmetrical model in which the galaxy has a radius of $R=1.75$ '. In this region, the background in the $0.3-2 \mathrm{keV}$ band has a mean of 16.3 counts, and we thus place the $99 \%$ confidence upper limit at 27 total counts (or 10.7 source counts), following the method described in $\S$ 2.1.1. Using the $\beta$ model (Equation 2) into Equation 1, we obtain upper limits to the central gas density:

$$
n_{e 0}=\left(\frac{K 10^{14} 4 \pi D^{2}}{4 \pi r_{c}^{3} I_{1}\left(R / r_{c}\right)}\right)^{\frac{1}{2}}
$$

in which the integration of the density profile has led to the integral $I_{1}\left(R / r_{c}\right)=\int_{0}^{R / r_{c}} x^{2}(1+$ $\left.x^{2}\right)^{-3 \beta} d x$. We use two reference values for the index $\beta$ : a value of $\beta=2 / 3$, as usually measured in galaxy clusters (e.g., LaRoque et al. 2006), and a shallower slope of $\beta=1 / 3$, which may be typical of lower-mass halos (e.g., galaxy groups and galaxies). We find analytical solutions for the integral, $I_{1}(n)=n /\left(2+2 n^{2}\right)+\arctan (n) / 2$ for $\beta=2 / 3$, and $I_{1}(n)=n-\arctan (n)$ for $\beta=1 / 3$.

We then estimate the upper limits to the gas mass by integration of the density profile,

$$
\begin{aligned}
M_{\text {gas }} & =\mu_{e} m_{H} \int_{0}^{R} n_{e 0}\left(1+\frac{r^{2}}{r_{c}^{2}}\right)^{-\frac{3}{2} \beta} 4 \pi r^{2} d r \\
& =4 \pi \mu_{e} m_{H} n_{e 0} r_{c}^{3} I_{2}\left(R / r_{c}\right)
\end{aligned}
$$

in which the $\beta$ model profile has led to the integral $I_{2}\left(R / r_{c}\right)=\int_{0}^{R / r_{c}} x^{2}\left(1+x^{2}\right)^{-3 \beta / 2} d x$. This integral is $I_{2}(n)=n-\arctan (n)$ for $\beta=2 / 3$, and $I_{2}(n)=\ln \left(x+\sqrt{1+x^{2}}\right)$ for $\beta=1 / 3$. 
We estimate upper limits to the gas mass as function of plasma temperature using Equations 3 and 4, using a fiducial value of the core radius of 0.5' (Figure 2). We determine that the upper limits to the gas mass depend weakly on the choice of the core radius, given that varying the fiducial value by $\pm 100 \%$ results in variation of less than $10 \%$. This model results in upper limits to the gas mass that are in the range of $M_{\text {gas }} \simeq 4 \times 10^{7}-2 \times 10^{8}$ $M_{\odot}$. Upper limits to the gas mass are therefore similar to those obtained from the simple uniform density model of Section 2.1.1.

\subsection{Point source $\mathrm{X}$-ray emission}

We set upper limits to the luminosity of an unobserved point source anywhere within the $3.5^{\prime} \times 1.375^{\prime}$ region of interest. The upper limit takes into account that there are 68 statistically-independent SWIFT spatial resolution elements within this region, given that the half-power diameter of the XRT point spread function at $1.5 \mathrm{keV}$ is 18 ". The measured background, per 18"-diameter spatial resolution element is 0.131 , or 0.144 using a $+10 \%$ allowance for vignetting. Thus, considering the Poisson distribution with a mean of 0.144 and a total of 4 counts, there is a probability of only $1.6 \times 10^{-5}$ of measuring 4 or more counts in just one of these resolution elements. Of course we would have been happy to measure 4 or more counts in any one of the 68 elements so the joint probability is correspondingly larger. Thus, we may state, with approximately 99.9\%-confidence that we have not detected a a source of 4 counts or brighter from this region.

We consider a power-law emission model with photon index $\Gamma=2.0$ and determine the upper limits to the source luminosity as function of the absorbing Hi column density using XSPEC. The upper limits are shown in Figure 2, and are in the $L=3 \times 10^{38}-10^{40} \mathrm{ergs} \mathrm{s}^{-1}$ range. This upper limit corresponds to a flux of $8 \times 10^{-15} \mathrm{ergs} \mathrm{cm}^{-2} \mathrm{~s}^{-1}$ in the $0.3-2 \mathrm{keV}$ band.

\subsection{UV and IR emission}

For completeness, we also analyzed extant non-X-ray images of VIRGOHI 21.

Swift UVOT images using the UVM2 filter (effective wavelength $2231 \AA$ ) were obtained concurrently with the XRT data. No sources were detected within the angular extent of VIRGOHI 21. The FTOOLS utility uvotsource was used to estimate a limiting magnitude for the UV emission from the $3.5^{\prime} \times 1.375^{\prime}$ region enclosing VIRGOHI 21 . This limit does not improve our estimates of the upper limit to the mass of diffuse hot gas nor to the flux from 
a point source as estimated above from the X-ray data.

We also analyzed archival Spitzer data (Program ID 30725) which included all four IRAC and three MIPS spectral bands. Again, no extended emission was detected within the Hi extent of VIRGOHI 21. We estimate an upper limit to the mass of dust associated with VIRGOHI 21 to be $10^{4}-10^{6} M_{\odot}$ depending on the dust temperature assumed.

\section{Discussion and conclusions}

A search for X-ray emission from the Hi line source VIRGOHI 21 using the Swift XRT obtained only upper limits. The non-detection indicates that any diffuse X-ray emitting gas at $k T=0.1-1 \mathrm{keV}$ must be less massive than $4 \times 10^{7}-2 \times 10^{8} M_{\odot}$, depending on the gas temperature and its spatial distribution. Given that the Swift XRT detector has no effective area approximately below $0.1 \mathrm{keV}$, it is possible that significant amounts of gas at sub-virial temperature have gone undetected by this X-ray observation. Similarly, any X-ray point source associated with VIRGOHI 21 will have a luminosity lower than $5 \times 10^{38}-10^{40}$ $\operatorname{ergs~} \mathrm{s}^{-1}$, depending on the absorbing column density of gas along the line of sight.

How do these limits compare with expectations? There are well-established correlations between optical and X-ray light from galaxies: Typical $f_{\mathrm{X}} / f_{O}$ ratios for normal galaxies range from $10^{-4}$ to $10^{-2}$ and for galaxies hosting AGNs the range extends up to unity (e.g., Kim et al. 2006). For the dark galaxy VIRGOHI 21, these ratios predict an X-ray flux of $10^{-17}$ to $10^{-15}$ for a normal galaxy up to $10^{-13}$ if an AGN is present. Of course, these $f_{\mathrm{X}} / f_{O}$ correlations reflect the causal link between stellar processes and X-ray light. They are not expected to hold in the absence of stars.

Instead, our expectation that VIRGOHI 21 should be a source of diffuse X-ray emission stems from the fundamental picture (Rees \& Ostriker 1977) that gas falling into a DM halo is first heated to the virial temperature. The accumulation of hot gas over a Hubble time could be substantial for a massive DM halo like VIRGOHI 21 (White \& Frenk 1991). We would expect a gas virial temperature of approximately $0.4 \mathrm{keV}$ based on the observed kinematics of VIRGOHI 21 (from Minchin et al. 2007) and thus our best guess to the amount of hot gas associated with VIRGOHI 21 is $\leq 5 \times 10^{7} M_{\odot}$. Scaling from simulations of gas infall onto stable disk galaxies (Benson et al. 2000; Toft et al. 2002), the X-ray luminosity of this gas should be up to $5 \times 10^{38} \mathrm{ergs} \mathrm{s}^{-1}$ for rotation speeds similar to that of VIRGOHI 21 .

The above estimates are based on the assumption of an isolated 'quiescent' massive galaxy accreting slowly (i.e., with a long cooling time) from the surrounding intergalactic medium. In the particular case of VIRGOHI 21, in contrast, a considerable gas reservoir 
exists within the tidal tail emanating from NGC 4254 that should lead to a relatively high gas accretion rate and hence high X-ray luminosity. Furthermore, turbulence and shocks following the interaction between VIRGOHI 21 and NGC 4254 would very likely heat some portion of the disk HI gas component of VIRGOHI 21 to X-ray emitting temperatures. We therefore consider the above estimates to be minimal expectations. Of course, any prediction

of the amount of hot gas or its luminosity will scale with the dynamical mass of the accreting object.

The observed upper limits reported here, therefore, show that the extreme object VIRGOHI 21 is not an extreme X-ray object (in the sense of a high $f_{\mathrm{X}} / f_{O}$ ) nor has it accreted very much gas from its surroundings during its lifetime.

\section{REFERENCES}

Bekki, K., Koribalski, B. S., \& Kilborn, V. A. 2005, MNRAS, 363, L21

Benson, A. J., Bower, R. G., Frenk, C. S., Lacey, C. G., Baugh, C. M., \& Cole, S. 2003, ApJ, 599, 38

Benson, A. J., Bower, R. G., Frenk, C. S., \& White, S. D. M. 2000, MNRAS, 314, 557

Blanton, M. R. et al. 2001, AJ, 121, 2358

Cavaliere, A., \& Fusco-Femiano, R. 1978, A\&A, 70, 677

Cusumano, G. et al. 2006, Memorie della Societa Astronomica Italiana Supplement, 9, 358

Davies, J. et al. 2004, MNRAS, 349, 922

Davies, J. I., Disney, M. J., Minchin, R. F., Auld, R., \& Smith, R. 2006, MNRAS, 368, 1479

Dickey, J. M., \& Lockman, F. J. 1990, ARA\&A, 28, 215

Doyle, M. T. et al. 2005, MNRAS, 361, 34

Duc, P.-A., \& Bournaud, F. 2008, ApJ, 673, 787

Haynes, M. P., Giovanelli, R., \& Kent, B. R. 2007, ApJ, 665, L19

Kalberla, P. M. W., Burton, W. B., Hartmann, D., Arnal, E. M., Bajaja, E., Morras, R., \& Pöppel, W. G. L. 2005, A\&A, 440, 775

Kim, D.-W., et al. 2006, ApJ, 644, 829 
Klypin, A., Kravtsov, A. V., Valenzuela, O., \& Prada, F. 1999, ApJ, 522, 82

LaRoque, S. J., Bonamente, M., Carlstrom, J. E., Joy, M. K., Nagai, D., Reese, E. D., \& Dawson, K. S. 2006, ApJ, 652, 917

Mateo, M. L. 1998, ARA\&A, 36, 435

McKee, C. F., \& Ostriker, J. P. 1977, ApJ, 218, 148

Mei, S. et al. 2007, ApJ, 655, 144

Minchin, R. et al. 2005, ApJ, 622, L21

-. 2007, ApJ, 670, 1056

Moore, B., Ghigna, S., Governato, F., Lake, G., Quinn, T., Stadel, J., \& Tozzi, P. 1999, ApJ, 524, L19

Morrison, R., \& McCammon, D. 1983, ApJ, 270, 119

Rees, M. J., \& Ostriker, J. P. 1977, MNRAS, 179, 541

Romano, P. et al. 2005, in Presented at the Society of Photo-Optical Instrumentation Engineers (SPIE) Conference, Vol. 5898, UV, X-Ray, and Gamma-Ray Space Instrumentation for Astronomy XIV. Edited by Siegmund, Oswald H. W. Proceedings of the SPIE, Volume 5898, pp. 369-376 (2005)., ed. O. H. W. Siegmund, 369-376

Spitzer, L. J. 1956, ApJ, 124, 20

Tagliaferri, G. et al. 2004, in Presented at the Society of Photo-Optical Instrumentation Engineers (SPIE) Conference, Vol. 5165, X-Ray and Gamma-Ray Instrumentation for Astronomy XIII. Edited by Flanagan, Kathryn A.; Siegmund, Oswald H. W. Proceedings of the SPIE, Volume 5165, pp. 241-250 (2004)., ed. K. A. Flanagan \& O. H. W. Siegmund, 241-250

Toft, S., Rasmussen, J., Sommer-Larsen, J., \& Pedersen, K. 2002, MNRAS, 335, 799

Vollmer, B., Huchtmeier, W., \& van Driel, W. 2005, A\&A, 439, 921

Wang, Q. D. 2005, in Astronomical Society of the Pacific Conference Series, Vol. 331, ExtraPlanar Gas, ed. R. Braun, 329-+

White, S. D. M., \& Frenk, C. S. 1991, ApJ, 379, 52

White, S. D. M., \& Rees, M. J. 1978, MNRAS, 183, 341 
This preprint was prepared with the AAS LATEX macros v5.2. 

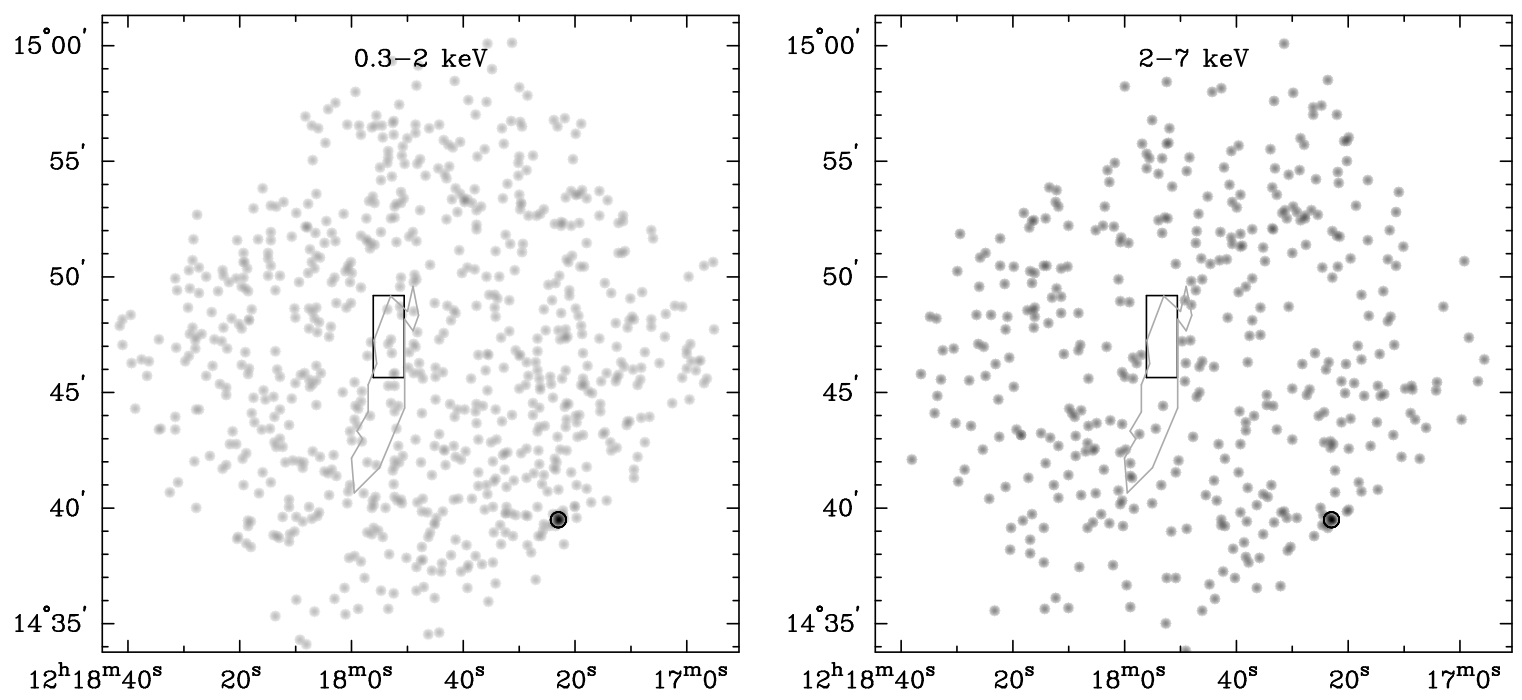

Fig. 1. - Swift XRT images of VIRGOHI 21, smoothed with a Gaussian kernel of $\sigma=7$ ". Grey contours are reproduced from the Minchin et al. (2007) Hi observations with the Westerbork telescope. The black box represent the location of the putative Hi rotating disc. The circle centered at $\mathrm{RA}=12^{h} 17^{m} 23^{s}, \delta=14^{\circ} 39^{\prime} 29^{\prime \prime}$ represents the region from which we extracted the counts of the serendipitous source SWIFT-XRT J121723+1439.5. 


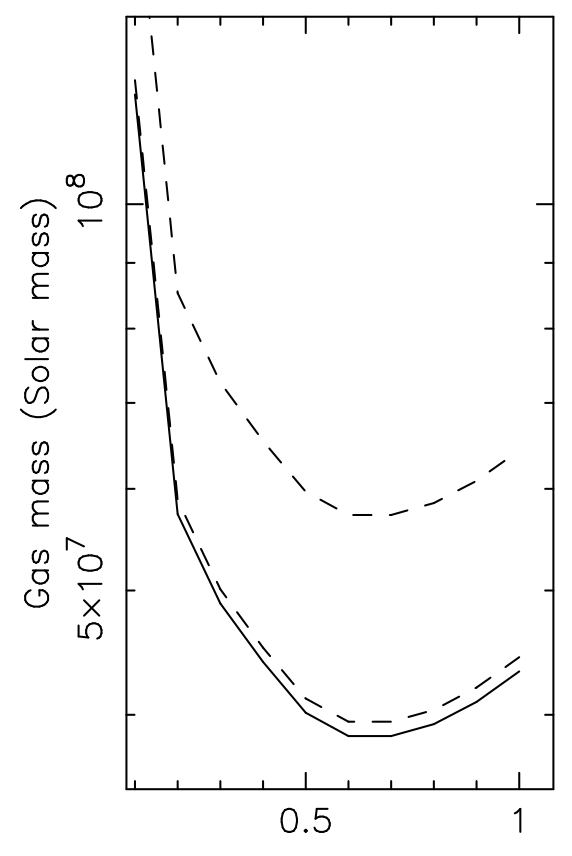

Gas temperature (keV)

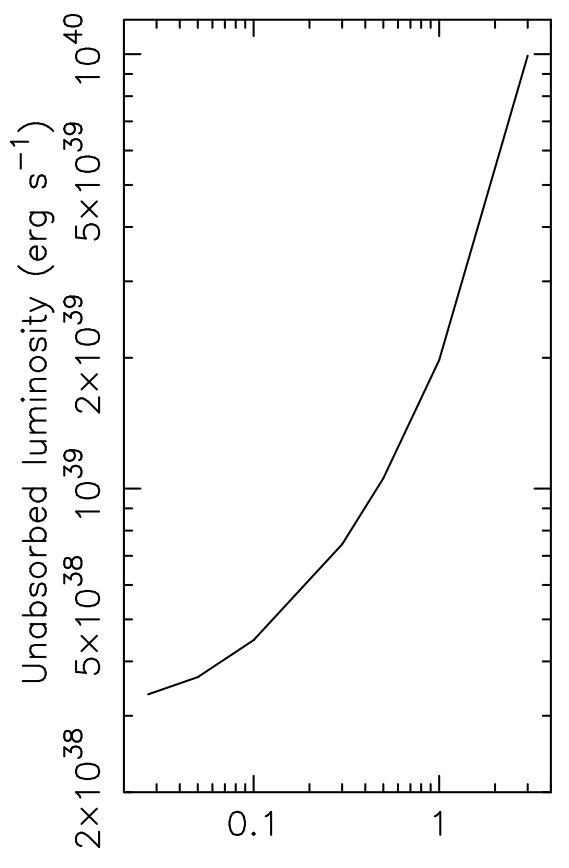

HI column $\left(10^{20} \mathrm{~cm}^{-2}\right)$

Fig. 2.- (a): Upper limits to the mass of diffuse gas in VIRGOHI 21, estimated assuming a detection threshold of 17 counts in these XRT observations. The Swift XRT bandpass is not sensitive to emission from diffuse gas at temperature $k T \leq 0.1 \mathrm{keV}$. Solid line : uniform density model, using a detection threshold of 8.2 counts in a $3.5^{\prime} \times 1.375$ ' box. Dashed lines : $\beta$ model with $r_{c}=0.5^{\prime}$, using a detection threshold of 10.7 counts in a circle of radius 1.75' (bottom line: $\beta=0.67$; top line: $\beta=0.33$ ). Given that the gas mass is proportional to the square root of the number of detected counts, the estimates are not very sensitive to changes in the detection threshold. (b) Upper limits to the luminosity of point sources in the VIRGOHI 21 field, estimated assuming a detection threshold of 4 source counts. The luminosity is proportional to the number of detected counts. 